\title{
¿Perdió el Área Metropolitana del Gran Santiago su atractivo? Sí, pero no. Un examen basado en datos y procedimientos novedosos para la estimación de la migración interna y sus efectos durante el periodo 1977-2013
}

Jorge Rodríguez. Comisión Económica para América Latina y el Caribe (CEPAL), Santiago, Chile.

Katherine Páez. Comisión Económica para América Latina y el Caribe (CEPAL), Santiago, Chile.

Cristóbal Abarca. Comisión Económica para América Latina y el Caribe (CEPAL), Santiago, Chile.

Ignacio Becker. Comisión Económica para América Latina y el Caribe (CEPAL), Santiago, Chile.

RESUMEN | La migración interna tiene efectos cuantitativos y cualitativos sobre el Área Metropolitana del Gran Santiago (AMGs). Estos efectos pueden influir sobre la evolución demográfica y socioeconómica del AMGs, evolución sobre la cual hay hipótesis académicas y posiciones políticas encontradas. Para estimar estos efectos, en este estudio se utilizan los últimos censos del país, salvo el de 2012, y las encuestas de Caracterización Socioeconómica Nacional (CASEN), así como nuevas metodologías. Los resultados muestran la paulatina pérdida de atractivo del AMGS, aunque al mismo tiempo revelan que esta tendencia tiene irregularidades y depende parcialmente de la definición geográfica del AMGs. Los resultados también evidencian que los efectos de la migración interna sobre la composición sociodemográfica del AMGs son favorables para el Área Metropolitana, porque la migración la rejuvenece y eleva su nivel educativo, lo que contribuye a reforzar la posición dominante de la ciudad dentro del sistema de ciudades.

PALABRAS CLAVE | metropolización, migración, transformaciones socioterritoriales.

ABSTRACT | Internal migration has quantitative and qualitative effects on the Metropolitan Area of Greater Santiago (MAGs). These effects can influence the demographic and socio-economic development of MAGs, of which academic hypotheses and political arguments have been found. To estimate these effects, the authors used the latest censuses-with the exception of year 2012-and CASEN (Socio-economic Characterization) surveys, as well as new methodologies. The results show a gradual loss of attractiveness of MAGs, although they also reveal that this trend has irregularities and partially depends on the geographic definition of the MAGs. The results also show that the effects of internal migration on the socio-demographic composition of MAGS are beneficial for the metropolis because it rejuvenates and increases the educational level of the population, which contributes to strengthen the dominant position of MAGS within the network of cities.

KEYWORDS | metropolization, migration, socio-territorial transformations.

Recibido el 4 de febrero de 2016, aprobado el 4 de abril de 2016

E-mail: J. Rodríguez, jorge.rodriguez@cepal.org | K. Paez, kapaez@gmail.com | C. Abarca, cristobalabarcabrown@gmail.com | I. Becker, ibecker1@uc.cl 


\section{Introducción:}

\section{La migración interna y su relevancia demográfica}

La migración ${ }^{1}$ tiene varios efectos demográficos directos, entre los cuales sobresalen: (i) el que ejerce sobre el crecimiento de la población (efecto crecimiento), que es el más tradicional y conocido de todos; (ii) el que incide sobre la composición y las características de la población (efecto composición); (iii) el que opera sobre la distribución de la población (efecto distribución); y (iv) el que genera sobre la distribución en el territorio de los diferentes grupos sociales expuestos a migración (efecto segregación) (Bell \& Salut, 2009; Comisión Económica para América Latina y el Caribe [CEPAL], 2012; Rodríguez, 2013a). Cabe destacar que la migración no es el único causante de estos efectos, ya que también son producidos por los diferenciales territoriales del balance entre nacimientos y defunciones (crecimiento vegetativo o natural) y otros procesos, como la anexión de localidades. Cualquiera sea el caso, estimar el efecto específico y directo de la migración sobre el crecimiento, la composición, la distribución y la segregación territorial de la población es un aporte para la mejor comprensión del papel que desempeña la migración en las transformaciones demográficas a escala subnacional.

En el caso de Chile, diversas razones justifican una investigación sobre los efectos de la migración en la evolución demográfica del Área Metropolitana del Gran Santiago (AMGs). ${ }^{2}$ En primer lugar, de acuerdo con las proyecciones demográficas más recientes del Instituto Nacional de Estadísticas (INE), ${ }^{3}$ el AMGs concentra el 35\% de la población nacional, por lo cual su evolución demográfica incide decisivamente en la del país. En segundo lugar, una fracción significativa de las capacidades productivas, creativas y resolutivas que tiene el país también se concentran allí (De Mattos \& Hidalgo, 2007; Dureau, Lulle, Souchaud \& Contreras, 2014; Fuentes \& Sierralta, 2014; Galetovic \& Jordán, 2006), lo que refuerza su importancia a escala nacional. Y en tercer lugar, el papel del AMGS y su relación con el desarrollo nacional son objeto de un debate intenso, el cual tiene al menos cinco vertientes conceptuales.

En primer lugar, están las discusiones respecto de la concentración territorial de la población, y del capital en todas sus formas, que se da en el capitalismo, y en particular en su modalidad posfordista (Amin, 2001; De Mattos, 2010; Henderson, 2003; Pacione, 2009). En segundo lugar, la emergencia de nuevas

1 La migración en general, aunque en este texto, por razones sustantivas y metodológicas que se explican más adelante, se examina solo la migración interna, es decir, la que acontece entre divisiones político-administrativas de un mismo país.

2 Se examinan 34 comunas de las 47 de la Región Metropolitana de Santiago. Se trata de las 32 comunas de la provincia de Santiago más las comunas de Puente Alto, de la Provincia de Cordillera, y la comuna de San Bernardo, de la Provincia de Maipo. Esta definición, la más tradicional, no está ajena a polémicas (Dureau et al., 2014; Galetovic \& Jordán, 2006; Rodríguez \& Espinoza, 2012). En efecto, esta definición captura el grueso, si acaso no la totalidad, de la superficie urbana continua de la ciudad, pero pierde zonas de la Región Metropolitana de Santiago total o funcionalmente integradas a la ciudad, pese a existir solución de continuidad con la mancha urbana principal. Este trabajo intenta incorporar estas zonas mediante una clasificación especial del resto de las comunas de la Región Metropolitana de Santiago.

3 Véase http://www.ine.cl/canales/chile_estadistico/familias/demograficas_vitales.php 
formas metropolitanas (Brenner, 2013; Ingram, 1998; Organisation for Economic Co-operation and Development [OECD], 2012; Sassen, 2007), en particular el reemplazo de las tradicionales configuraciones concentradas por otras más difusas y de mayor alcance geográfico. En tercer lugar, las dudas sobre el impacto territorial, y en particular la incidencia sobre la concentración demográfica y económica en el AMGS, de la estrategia de desarrollo que privilegia la explotación y exportación de recursos naturales (Cunha \& Rodríguez, 2010; Daher, 2003; Rodríguez \& Espinoza, 2012; Rodríguez \& González, 2006). En cuarto lugar están los debates sobre los efectos de las políticas de desarrollo regional, de ordenamiento territorial, de desarrollo urbano y de descentralización sobre la histórica primacía metropolitana de Chile, y en la mayoría de los otros países de América Latina (Atienza \& Aroca, 2012; Cepal, 2012, 2015; Rodríguez \& Espinoza, 2012). Y en quinto lugar, las discusiones entre las posiciones que rechazan la concentración de recursos y oportunidades en el AMGS, por injusta e ineficiente (Atienza \& Aroca, 2012), y aquellas que la aceptan como una necesidad para el desarrollo nacional, como un resultado natural de las fuerzas del mercado o como un proceso histórico difícil de revertir, a menos que sea por las mismas fuerzas del mercado (Galetovic \& Jordán, 2006; Ingram, 1998).

Una dimensión consustancial a estos debates sobre la concentración y sobre el presente y el futuro del AMGS refiere a la migración, ya que esta puede reforzar o erosionar la concentración demográfica en ella. Y en línea con lo expuesto anteriormente sobre los diferentes efectos de la migración, es claro que la relación entre migración y centralismo no puede limitarse al efecto crecimiento, ya que el efecto composición -por ejemplo mediante el atractivo selectivo de personas con características más valoradas- puede ser tanto o más relevante para el reforzamiento del AMGs.

Lamentablemente, la investigación empírica sobre estos efectos en el AMGs ha sido escasa y en el último tiempo las posibilidades de revertir tal deficiencia se deterioraron dramáticamente con el fallido censo de 2012. Lo anterior, porque los datos censales son la única fuente para medir migración de manera detallada en Chile, debido a que no existen registros continuos de residencia.

Con todo, la importancia del tema obliga a buscar otras fuentes para evitar un periodo de quince años de "ceguera migratoria". Justamente en esa línea avanza esta investigación, la cual procura responder dos grandes preguntas usando microdatos censales de 1982 a 2002 y las encuestas de Caracterización Socioeconómica Nacional (CASEN), que desde 2006 incluyen un módulo de migración similar al del censo. Estas preguntas son: (i) ¿cómo ha evolucionado el efecto crecimiento de la migración sobre el AMGs en las últimas cuatro décadas?; y (ii) ¿cómo ha evolucionado el efecto composición de la migración sobre el AMGS en las últimas cuatro décadas?

El documento se estructura en cuatro secciones adicionales a esta introducción. A continuación se presenta un marco de referencia que incluye antecedentes, conceptos y relaciones conceptuales, y las hipótesis. Luego el marco metodológico, en el que se especifican los efectos que se medirán y se describen las fuentes, las variables y los procedimientos usados para obtener los resultados. Posteriormente, se exponen y analizan los resultados obtenidos para evaluar empíricamente las hipótesis. Finalmente, se plantean las conclusiones, se discuten sus alcances y perspectivas, se 
reflexiona sobre sus implicaciones de política y se llama la atención sobre los desafíos futuros en materia de investigación.

\section{Concentración y atractivo migratorio de las grandes ciudades: debate y evidencia}

Entre las migraciones internas más estudiadas en América Latina están las de las áreas metropolitanas (Alberts, 1977; Camisa, 1972; Chávez et al., 2013; CEPAL, 2012; Cunha \& Rodríguez, 2010; Elizaga, 1970, 1972; Elizaga \& Macisco, 1975; Rodríguez \& Busso, 2009; Villa \& Rodríguez, 1997). Durante el siglo xx lo fueron porque las áreas metropolitanas concentraron el atractivo migratorio $\mathrm{y}$, al mismo tiempo, fueron los espacios privilegiados tanto de la inversión pública y privada como del desarrollo económico y social. De hecho, en América Latina la urbanización, empujada por la transferencia de población del campo a las ciudades, fue de la mano con la metropolización, llevando a que a fines del siglo pasado uno de cada tres latinoamericanos viviera en una ciudad de más de 1 millón de habitantes (CEPAL, 2012) y que en la actualidad esa cifra llegue al 36\% (United Nations, 2015, pp. 38 y 84). Si bien el círculo virtuoso entre urbanización, desarrollo económico y progreso social que se experimentó en los países desarrollados no se repitió en los mismos términos en América Latina (CEPAL, 2012; Fondo de Población de las Naciones Unidas [UNFPA], 2007; Glaeser, 2011; Henderson, 2003; Martine, McGranahan, Montgomery \& Fernández-Castilla, 2008), es manifiesto que las grandes ciudades concentraron recursos, oportunidades y poder, lo que reforzó su posición dominante a escala nacional, y simultáneamente acumularon déficits y problemas por su rápido crecimiento, las dificultades para enfrentarlo, las incapacidades políticas e institucionales y la profunda desigualdad económica y social (CEPAL, 2012, 2015; Gilbert, 1996; Glaeser, 2011; Hall, 1996).

En este cuadro se enmarca la reversión del atractivo migratorio que han experimentado algunas áreas metropolitanas de América Latina, las que se han convertido en expulsoras, como Ciudad de México, São Paulo y Río de Janeiro. Las explicaciones que se han dado para esta reversión son diversas. Está la clásica hipótesis de Williamson (1965), que establece una relación de U invertida entre concentración y desarrollo económico. Está la de la autodenominada Nueva Geografía Económica (Fujita, Krugman \& Venables, 2000), que formaliza la dinámica económica territorial en términos de un juego de fuerzas centrípetas y centrífugas, cuyo balance puede conducir a la dispersión geográfica de la producción (aunque también puede llevar hacia su concentración, incluso sostenible). Por otro lado, también está la tesis que resalta el desarrollo tecnológico y el cambio en el paradigma productivo -el advenimiento del posfordismo con modalidades productivas más flexibles y dispersas a escala mundial-, que también fomentan la relocalización y dispersión de las actividades económicas y de la población (Amin, 2001). Está la que sugiere que las deseconomías de aglomeración y la competencia entre nodos del sistema de ciudades generan transiciones que implican desconcentración hacia ciudades medias y menores (Galetovic \& Jordán, 2006; Henderson, 2003; Ingram, 1998) o la que levanta la contraurbanización como reacción a los problemas generados 
en las grandes ciudades mediante un retorno a ciudades más pequeñas e incluso al campo (Berg, Drewett, Klaasen, Rossi \& Vijverberg, 1982; Geyer \& Kontuly, 1993; Greenwood, 1997; Hall, 1996; Pacione, 2009; United Nations, 2008).

Ahora bien, esta reversión ha sido cuestionada por varios enfoques. Uno de ellos levanta la hipótesis de la "desconcentración concentrada", según la cual la pérdida de gravitación demográfica (y también económica) del área metropolitana se debe a una ganancia de su entorno y, por tanto, se trata en realidad de una ampliación de la escala geográfica del área metropolitana (Campolina, 1994; CEPAL, 2012; Cunha, 2015; Cunha \& Rodríguez, 2010; De Mattos, 2010; Sabatini, 1991). Un enfoque alternativo esgrime la hipótesis de la ciudad-región, donde lo que habría en realidad no sería una pérdida de atractivo de la ciudad, sino una ampliación de su escala en términos de una constitución plurinodal mediante la articulación con un conjunto de ciudades vecinas (Sassen, 2007). Otros, por su parte, reiteran la transitoriedad de la reversión por las inclemencias pasajeras que vivieron las grandes ciudades en las décadas finales del siglo xx (CEPAL, 2012; Rodríguez \& Espinoza, 2012). También están las miradas evolucionistas, como los enfoques de transición urbana o metropolitana, que incluyen una etapa final de reconcentración o recuperación metropolitana (Cunha, 2015; Pacione, 2009). Finalmente, cabe mencionar el enfoque de la ciudad global, que subrayó las nuevas funciones de las grandes ciudades en la economía globalizada (De Mattos, 2010; Sassen, 1991).

Habida cuenta de estos debates, no es extraño que actualmente haya serias dudas sobre las expectativas desconcentradoras abiertas en la región, y en particular en Chile, por el cambio de modelo de desarrollo, las nuevas modalidades productivas, la revolución tecnológica y las iniciativas descentralizadoras (Daher, 2003; Rodríguez \& Espinoza, 2012; Sabatini \& Wormald, 2008). En esa línea, un trabajo reciente concluye que "a pesar del crecimiento experimentado por Chile durante las últimas décadas, la fuerte primacía de la Región Metropolitana se mantiene con firmeza” (Atienza \& Aroca, 2012, p. 272), debido a varios factores, entre los cuales está la pertinaz concentración en el AMGs de las instituciones de innovación y conocimiento, como universidades, centros de estudio y núcleos de investigación.

Una dimensión fundamental de este debate sobre la tendencia y el futuro de la concentración demográfica y económica del AMGs y sobre su gravitación en el desarrollo nacional, atañe a la migración. Su "efecto crecimiento" -es decir, su impacto sobre el crecimiento de la población- ya no puede darse por sentado, teniendo presente la inflexión del atractivo de las grandes ciudades recién comentada. Y lo mismo acontece con su efecto composición, es decir, su impacto sobre las características de la población. Cuando la inmigración a las grandes ciudades provenía principalmente de zonas rurales o de ciudades pequeńas y carenciadas, tenía una sobrerrepresentación de jóvenes en busca de trabajo y estudio; de mujeres en busca de opciones vitales, y en particular laborales, que eran escasas en sus lugares de origen; y de personas con escasa educación, por la falta de opciones educativas en los lugares de donde provenían (Alberts, 1977; Camisa, 1972; Elizaga, 1970; Elizaga \& Macisco, 1975). Por lo anterior, los efectos de composición de la migración tendían a ser predecibles, aunque nunca se les haya estimado con precisión por falta de metodologías adecuadas; más concretamente, producían una feminización, 
rejuvenecimiento (no en el sentido demográfico de aumentar la población infantil, sino en el literal de aumentar la proporción de jóvenes) y reducción del nivel educativo de las grandes ciudades (Rodríguez, 2013a, 2013b). Pero el agotamiento de la migración desde el campo y el entramado más complejo y diverso del sistema de ciudades llevan a que los flujos de inmigrantes, y también de los emigrantes, se comporten de una forma menos predecible, pudiendo tener efectos de composición diferentes a los del pasado, lo que se indagará empíricamente en este estudio.

Un punto importante de la discusión tiene que ver con los atractivos que tiene el AMGs. La mayor parte de la discusión sobre la desconcentración demográfica tiene como fundamento la desconcentración industrial, de manera tal que los afectados por ella serán, en primer lugar, los trabajadores de dicho sector. Pero esta desconcentración industrial podría darse con independencia de la que incide en otras actividades productivas, en línea con lo planteado por el enfoque de la ciudad global, que subraya el persistente atractivo de las grandes ciudades para el sector servicios y para el quehacer intelectual, cultural y universitario, como lo sugieren Atienza y Aroca (2012). Debido a lo anterior, pueden producirse simultáneamente procesos de desconcentración de ciertas actividades y de la población asociada a ellas, con la continuidad o incluso la acentuación de la concentración de otras actividades económicas y la población vinculada a ellas.

\section{Sobre las ventajas del AMGs: un primer acercamiento empírico}

Solo para evaluar si tiene validez empírica el debate anterior sobre la pertinaz ventaja del AMGs -factor clave para su eventual atractivo y selectividad migratoria, sobre todo si se trata de oportunidades educativas y laborales para los jóvenes, y de mejores niveles de vida-, se procesaron las bases de microdatos de los censos de 1982, 1992 y 2002 y las encuestas CASEN de 2006, 2009, 2011 y 2013, a fin de estimar la evolución de indicadores socioeconómicos seleccionados del AMGs respecto del total nacional. La figura 1 sintetiza los resultados, y las dos principales conclusiones que se pueden extraer son:

a. El AMGs sigue teniendo una situación mejor al promedio nacional en todos los indicadores examinados, entendiendo que "mejor" significa: (i) una relación superior a 1 entre el cociente del indicador AMGS y del indicador promedio nacional en los indicadores promedio de escolaridad, tasa de asistencia, tasa de participación y nivel de ingreso; (ii) una relación inferior a 1 entre el cociente del indicador AMGS y del indicador promedio nacional en los indicadores tasa de desempleo y porcentaje de pobreza.

b. En general, la brecha favorable al AMGs ha tendido a mantenerse o acentuarse, en algunos casos con oscilaciones. Incluso en el desempleo y la pobreza se ha ensanchado, en el caso del desempleo por el mayor nivel que registraba el AMGS en 1982, y en el caso de la pobreza por la acentuación de la brecha en el periodo 2011-2013.

En suma, se validan, en términos generales, las aseveraciones de Atienza y Aroca antes citadas y se fortalece la hipótesis que plantea la persistencia de las fuerzas de atracción del AmGs, tanto para la población total como para los jóvenes y los más 
escolarizados. Tal conclusión es respaldada por al menos uno de los pocos trabajos empíricos recientes sobre la migración interna del AMGS, que encuentra que tras la emigración neta del censo de 2002 y de la CASEN de 2006, el AMGS volvió a ser atractiva a fines de la década de 2000, en el marco de una fuerte crisis de algunas economías regionales, como la de la región de Los Lagos (Rodríguez \& Espinoza, 2012).

FIGURA I | AMGS: Relación con el promedio nacional en indicadores sociales seleccionados, 1982-2013

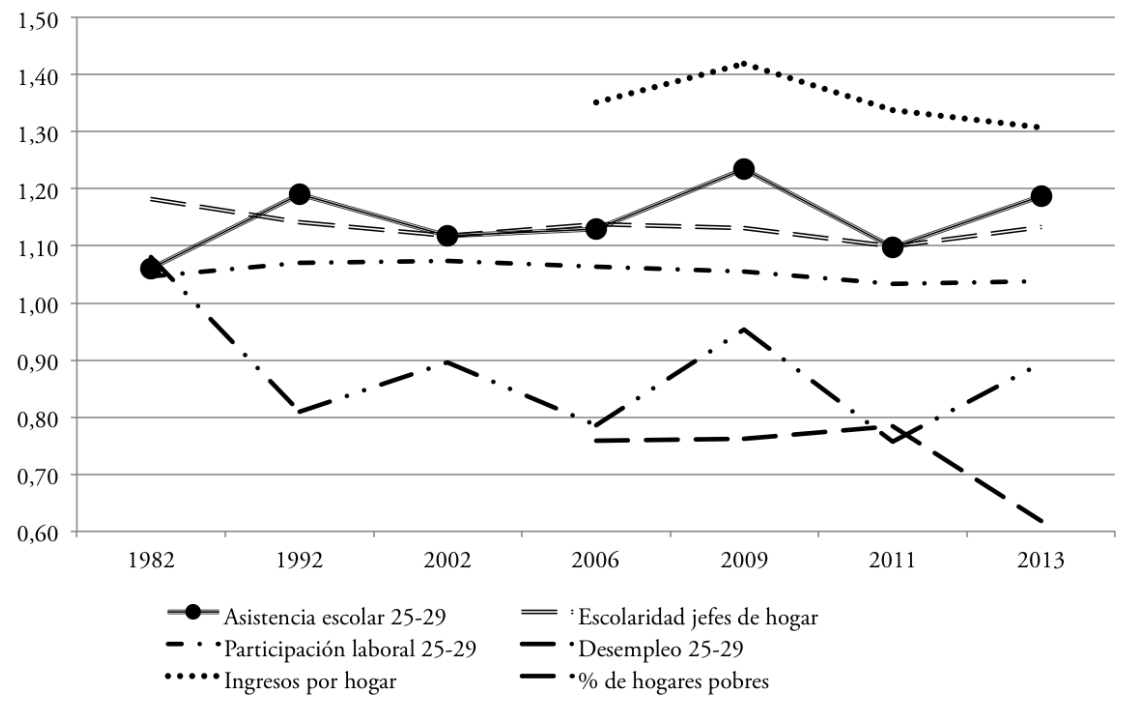

FUENTE ELABORACIÓN PROPIA A PARTIR DE PROCESAMIENTOS DE CENSOS: BASES DE MICRODATOS DE I982, I992 Y 2002; Y DE ENCUESTAS CASEN: BASES DE DATOS DE 2006, 2009, 20II Y 2013

NOTA LA RELACIÓN CORRESPONDE AL VALOR DEL INDICADOR DEL AMGS SOBRE EL VALOR DEL INDICADOR NACIONAL

Considerando los argumentos, literatura y evidencia previa, en este documento se examinan las siguientes hipótesis sobre el atractivo (efecto crecimiento) y la selectividad (efecto composición) de la migración del AMGs:

a. Considerando la evidencia más reciente sobre una recuperación de la fortaleza productiva del AMGs y la persistencia de sus ventajas comparativas respecto del resto del país, el atractivo migratorio de esta área debiera persistir, al menos en relación con el resto de las regiones.

b. Considerando que el patrón dominante de migración de las ciudades ya no es del tipo rural-urbano, sino urbano-urbano, y teniendo en cuenta el agotamiento en el AMGs de ciertos nichos de ocupación históricamente intensivos en mano de obra migrante femenina (servicios doméstico, textil, etcétera), el efecto feminizador de la migración debiera haberse reducido o hasta anulado o revertido. 
c. Considerando la evidencia más reciente sobre la concentración de oportunidades educativas y laborales para los jóvenes en el AMGs, el atractivo migratorio del AMGS para estos grupos debiera persistir.

d. Considerando que el patrón dominante de migración de las ciudades ya no es del tipo rural-urbano, sino urbano-urbano, y teniendo en cuenta la concentración de oportunidades educativas y laborales de alto nivel en el AMGs y los mayores costos que tiene la vida en el AMGS, la selectividad de la migración ya no debiera ser de personas con baja educación y, por ende, el efecto reductor de la educación de la migración debiera haberse atenuado y desaparecido.

\section{Medición del efecto de la migración sobre la composición sociodemográfica del AMGS}

Las fuentes de datos son las bases de microdatos de los censos de 1982, 1992, 2002 y de las encuestas CASEN de 2006, 2009, 2011 y 2013 (debidamente ponderadas). Estas bases son procesadas con Redatam para los censos y SPSS para las encuestas CASEN.

La identificación de los migrantes se realiza mediante la pregunta sobre residencia anterior en una fecha fija del pasado. Esta fecha es 5 años antes del censo o encuesta en todos los casos, menos la CASEN 2006, en que la fecha de referencia del pasado es la del censo de 2002.

El instrumento para estimar el efecto crecimiento de la migración es la matriz de migración clásica, con tres orígenes-destinos, a saber, AMGs, resto de la Región Metropolitana (RM) y resto del país. Estas tres zonas fueron construidas como recodificación de las variables 'comuna de residencia actual' (solo en el censo, porque la CASEN es de derecho) y 'comuna de residencia 5 años antes'.

El instrumento para estimar el efecto composición es la matriz de indicadores de flujos, usando la misma división territorial de la matriz previa (CEPAL, 2012; Rodríguez, 2013a; Rodríguez \& Busso, 2009).

$\mathrm{El}$ indicador del efecto crecimiento es la tasa de migración neta, que corresponde al cociente entre el saldo migratorio y la población expuesta al riesgo de migrar. Esta última corresponde al promedio de la población residente actual y de la población residente anterior ( 5 años antes, salvo CASEN 2006, por lo cual el tiempo de exposición al riesgo en este caso es de 4,5 años) en cada división usada en la matriz. Se multiplica por mil, como es el estándar en las tasas de los componentes de la dinámica demográfica.

El indicador del cambio de composición de la población debido exclusivamente a la migración corresponde a la diferencia entre el valor actual del atributo (factual) y el valor que hubiera existido en ausencia de migración (contrafactual). Los niveles factuales y contrafactuales corresponden a los marginales de la matriz de indicadores de flujo. El efecto total puede descomponerse en el efecto de la inmigración (factual menos diagonal o valor del atributo para los no migrantes) y el efecto de la emigración (o valor de la diagonal menos el contrafactual). Este indicador tiene una expresión relativa: cociente del efecto absoluto de la migración sobre el valor contrafactual. Indica cuánto cambió el atributo en cada zona por el efecto neto y exclusivo 
de la migración. Un valor de $100 \%$ indica que el atributo tiene un valor actual que es el doble del que hubiera tenido sin migración. Un valor de $-50 \%$ indica que el atributo en esa zona tiene un valor actual que es la mitad del que hubiera tenido sin migración. En el anexo técnico se expone con detalle la obtención de una de las matrices usadas en el texto, y los cálculos efectuados y resultados obtenidos a partir de ella para estimar el efecto de la migración sobre la proporción de jóvenes del AmGs.

\section{Resultados: pese a su emigración neta, el AMGS atrae jóvenes y personas de alto nivel educativo}

\section{Sobre el atractivo del AMGS}

La figura 2 presenta la evolución de las tasas medias anuales de migración neta desde 1977-1982 a 2008-2013.

En ambas piezas de evidencia se aprecia que el atractivo del AMGs tuvo una inflexión desde fines de la década de 1990, ya que desde el censo de 2002 (es decir, quinquenio 1987-1992) en adelante registra saldo y tasas migratorias negativas. Esta condición se vio interrumpida momentáneamente a fines de la década de 2000 (encuesta CASEN 2009), en el marco de una crisis económica severa que parece haber afectado más fuertemente a las economías regionales basadas en la explotación de minerales y productos agropecuarios, en particular la industria del salmón (Rodríguez \& Espinoza, 2012), aun cuando en la figura 1 no se evidencia una resiliencia específica del AMGS a esta crisis, salvo por el aumento de la brecha de ingreso per cápita a favor del AMGs.

FIGURA 2 | Tasas de migración neta (medias anuales por mil) 2013-2008; 2011 2006; 2009-2004; 2006-2002; 2002-1997; 1992-1987; 1982-1977. AMgs, población de 5 años y más

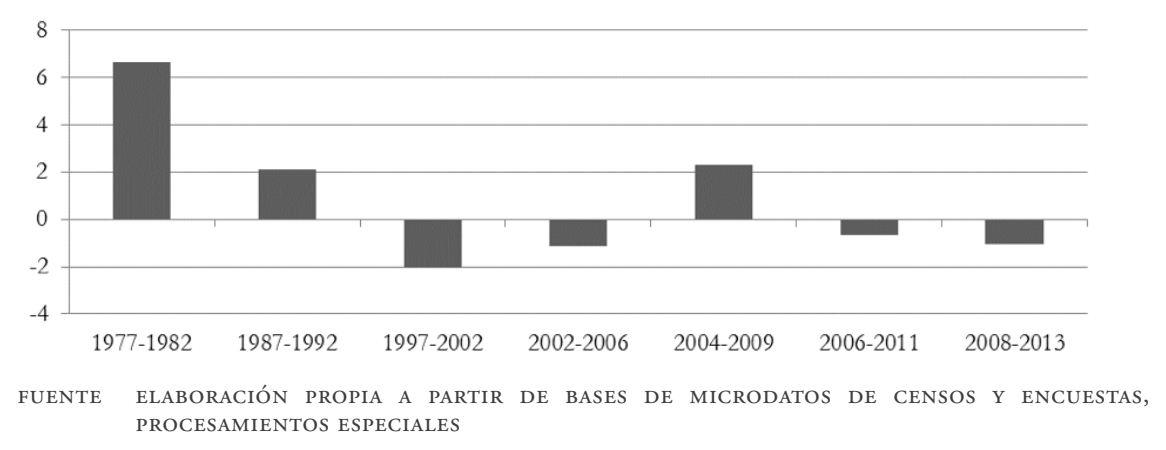

El balance migratorio entre el AMGS y el resto del país ofrece una primera pieza de evidencia para la discusión de fondo en que se enmarca esta investigación, que atañe a la concentración/desconcentración de la población chilena en su capital. De la figura 2 se deduce que en todos los casos desde 2002, con excepción de 2009, ese 
balance favorece al resto del país. ${ }^{4}$ Lo anterior respalda la hipótesis de la desconcentración demográfica. Con todo, esta conclusión amerita más análisis para saber si la ganancia migratoria del "resto del país" está centrada en zonas cercanas o en zonas lejanas al AMGs, lo que se hace más adelante.

\section{Sobre el atractivo migratorio del AMGs según sexo, edad y nivel educativo Según sexo}

La figura 3 muestra que hasta mediados de la década de 2000, el atractivo migratorio del AMGs seguía teniendo un sesgo femenino, por cuanto el saldo migratorio y las tasa de migración neta de las mujeres eran mayores que el saldo y las tasas de los hombres. Esto era válido incluso en lo referente a la emigración neta registrada por el censo de 2002 y la CASEN de 2006, pues en ambos el saldo y la tasa (ambos negativos) fueron menores en términos absolutos; es decir, el AMGs fue menos expulsora de mujeres que de hombres.

Ahora bien, este patrón ha tenido variaciones en los últimos años, tal como se plantea en las hipótesis. En efecto, la CASEN 2009 muestra un atractivo similar para ambos sexos (2,3 por mil), y sobre todo la CASEN 2013 muestra una mayor expulsión femenina (tasa de emigración neta de 1,5 por mil anual contra una de 0,6 de los hombres). ${ }^{5}$

Responder cabalmente a la pregunta sobre por qué actualmente el AMGs pareciera ser más atractiva para los hombres (o menos expulsora, en rigor) escapa a los alcances de este estudio, pues habría que indagar detenidamente en los factores de atracción y expulsión distintivos según sexo. Una línea de análisis que no se presenta aquí, pero que puede desarrollarse con apoyo en la metodología usada, es la distinción entre el efecto de la inmigración y el de la emigración. Si la inmigración continúa siendo una fuerza que feminiza el AMGs, entonces los factores de atracción históricos del AMGs para las mujeres -concretamente, su dinámico sector servicios, y en particular aquellos con una sobrerrepresentación femenina, como el servicio doméstico- mantendrían su imán migratorio para las mujeres. Pero si esto ya no es así, entonces habría que buscar en el mercado de trabajo de las ciudades la

Censo 1982: $232119-114218$ = 117901; Censo 1992: $210837-161514=49323$; Censo 2002: $200933-218758=-17825$; CASEN 2006: $108088-122997=-14909$; CASEN $2009=119284-$ 38383 = 80901; CASEN 2011: 145210 - 148341 = -3131; y CASEN 2013: 132736-139743 = -7007.

5 Cabe subrayar que estas diferencias de tasas ya anticipan el efecto de la migración sobre la composición por sexo del Amgs. Pero de la mera comparación de tasas no es posible calcular cuánto cambió la composición por sexo de la población por migración. Para estimar esto último se debe usar el procedimiento especial y novedoso explicado en el marco metodológico. En este sentido, no hay que confundir la selectividad de los flujos sugerida por el diferencial de tasas, por una parte, con la composición de los flujos, por otra. Para ilustrarlo con un ejemplo simple, pensemos en una zona en que solo hay inmigración. Un flujo mayoritariamente femenino no implicaría forzosamente una selectividad femenina del mismo respecto del destino. La selectividad femenina y, por ende, el efecto feminizador de la inmigración, solo ocurrirían si la relación de masculinidad del flujo de inmigrantes es MENOR que la relación de masculinidad de la población no migrantes. Entonces, si la relación de masculinidad de la población no migrante es 90 ( 90 hombres por cada por 100 mujeres), un flujo con un ligero predominio femenino, digamos una relación de masculinidad de 95, No sería selectivo de mujeres y en vez de feminizar el destino, lo masculinizaría. 
explicación del cambio, en particular en el decaimiento de las ocupaciones históricamente femeninas, como el servicio doméstico. ${ }^{6}$

FIGURA 3 | Tasas de migración neta (medias anuales por mil) por sexo, 20132008; 2011-2006; 2009-2004; 2006-2002; 2002-1997; 1992-1987; 1982-1977. AMGs, población de 5 años y más

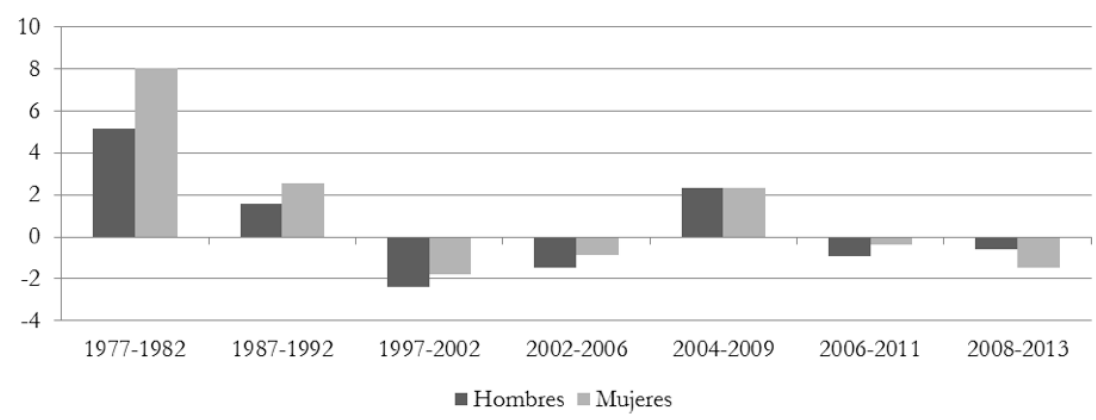

FUENTE ELABORACióN PROPIA A PARTIR DE BASES DE MICRODATOS DE CENSOS Y ENCUESTAS, PROCESAMIENTOS ESPECIALES

\section{Según edad}

En la figura 4 se aprecia claramente que hay diferencias significativas en el atractivo del AMGs según edad. El hecho estilizado más relevante es el persistente y significativo atractivo que tiene el AMGs para los jóvenes (15-29 años de edad), que sistemáticamente tienen tasas de migración neta mayores que el resto de los grupos, y durante todo el periodo de referencia la mantuvieron positiva.

En clara contraposición al atractivo que genera el AMGs para los jóvenes, para el resto de las edades el panorama es variopinto y en los últimos años ha estado marcado por la expulsión de los otros tres grupos de edad.

La expulsión de niños y adultos puede explicarse mediante un factor común, en la medida en que se trata de niños que emigran con adultos desde el AMGs. Estos casos de emigración familiar desde el AMGs, probablemente obedecen a razones de trabajo de los adultos en los casos de desplazamiento hacia el resto de las regiones del país, o procesos de suburbanización de clase media y alta y de asignación de vivienda social para clases más populares, en aquellos de desplazamientos hacia el resto del AMGs.

En el caso de las personas mayores, trabajos anteriores ya habían mostrado una salida desde el AMGs hacia zonas más acogedoras para este grupo de edad, concentradas en las regiones iv de Coquimbo y v de Valparaíso, sobre todo en el litoral (Rodríguez \& González, 2006). 
FIGURA 4 Tasas de migración neta (medias anuales por mil) por grandes grupos de edad, 2013-2008; 2011-2006; 2009-2004; 2006-2002; 20021997; 1992-1987; 1982-1977. AMGs, población de 5 años y más

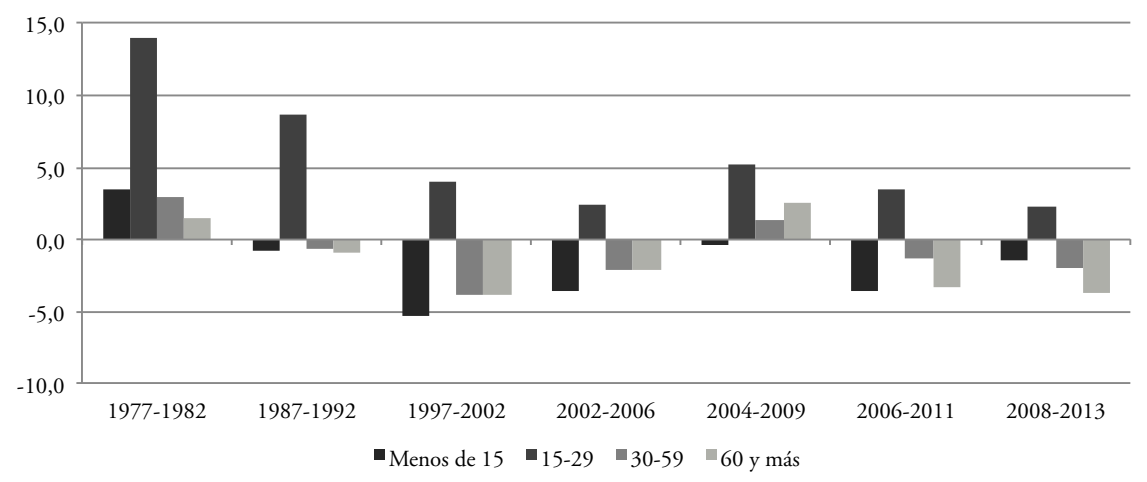

FUENTE ELABORACIÓN PROPIA A PARTIR DE BASES DE MICRODATOS DE CENSOS Y ENCUESTAS, PROCESAMIENTOS ESPECIALES

\section{Según educación}

En materia educativa, las figuras 5.1, 5.2 y 5.3 muestran el magnetismo que tiene el AMGs para las personas con mayor educación. Esto no era tan claro en el primer quinquenio analizado, toda vez que el AMGs resultaba atractiva para todos los grupos educativos, y de hecho las mayores tasas de inmigración neta no las registraban los universitarios, sino las personas con educación media y técnica superior. Pero desde fines de la década de 1980, los universitarios, y en particular los posgraduados (categoría que solo es posible de identificar con la CASEN), son los que registran las mayores tasas de inmigración neta; más aún, en los periodos más recientes son los únicos que registran migración neta positiva.

Este comportamiento marca una diferencia sustancial con la imagen histórica de una inmigración masiva a las grandes ciudades por parte de personas con baja educación. Se trata de una imagen anclada en la época del éxodo rural y del crecimiento explosivo de las ciudades. Y para muchos observadores, sigue siendo una imagen válida, en parte porque en diversas ciudades de América Latina los inmigrantes aún siguen teniendo una educación inferior a la de los no migrantes de estas ciudades. Pero las cifras dicen lo contrario para el AMGs, que en virtud de sus ventajas en materia de oferta educativa terciaria (y posterciaria), así como en el plano de empleos para personal de la mayor calificación, funciona como un "imán" para las personas con educación universitaria y posgrado. 
FIGURA 5.I | Tasas de migración neta (medias anuales por mil) por niveles educativos, 2013-2008; 2011-2006; 2009-2004; 2006-2002; 20021997; 1992-1987; 1982-1977. AMgs, población de 5 ańos y más

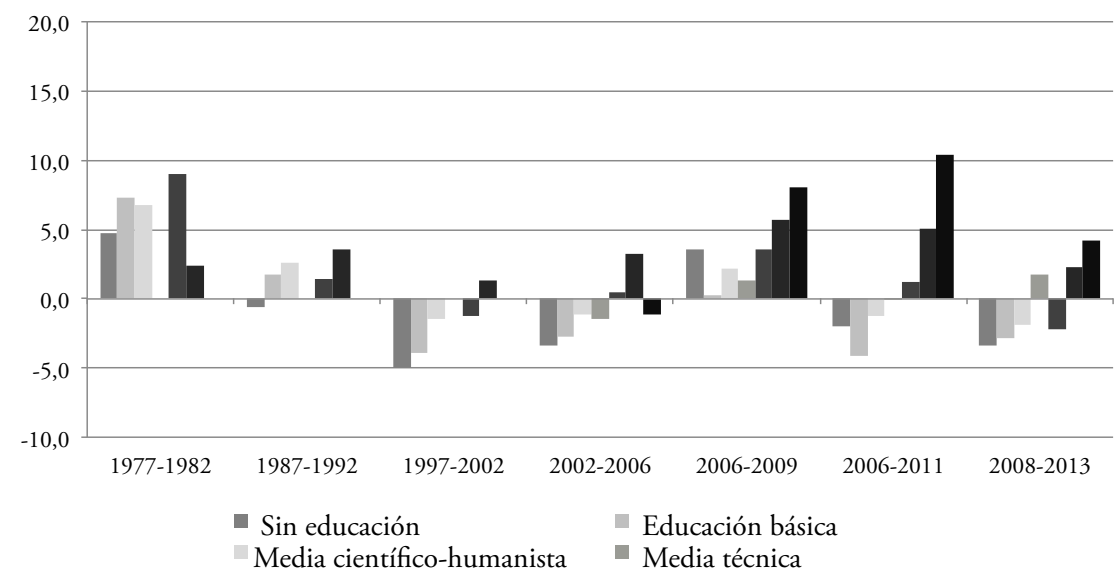

Fuente Elaboración propia a partir de bases de Microdatos de Censos y encuestas, PROCESAMIENTOS ESPECIALES

FIGURA 5.2 Tasas de migración neta (medias anuales por mil) por niveles educativos, 2013-2008; 2011-2006; 2009-2004; 2006-2002; 20021997; 1992-1987; 1982-1977. AMgs, población de 25 a 29 años

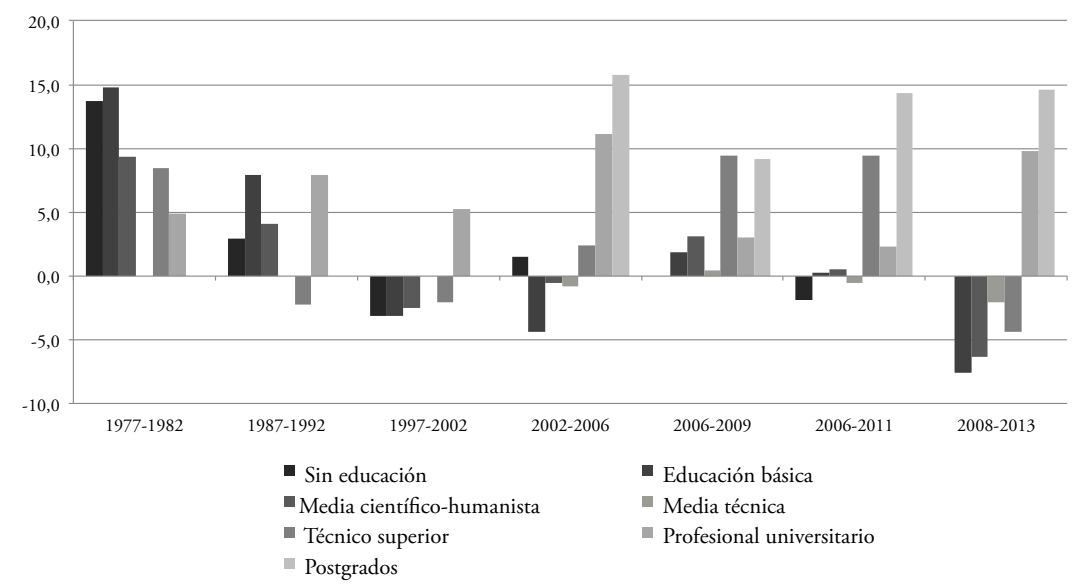

Fuente Elaboración propia a partir de bases de microdatos de Censos y encuestas, PROCESAMIENTOS ESPECIALES 
FIGURA 5.3 Tasas de migración neta (medias anuales por mil) por niveles educativos, 2013-2008; 2011-2006; 2009-2004; 2006-2002; 20021997; 1992-1987; 1982-1977. AMGs, población de 30-34 años

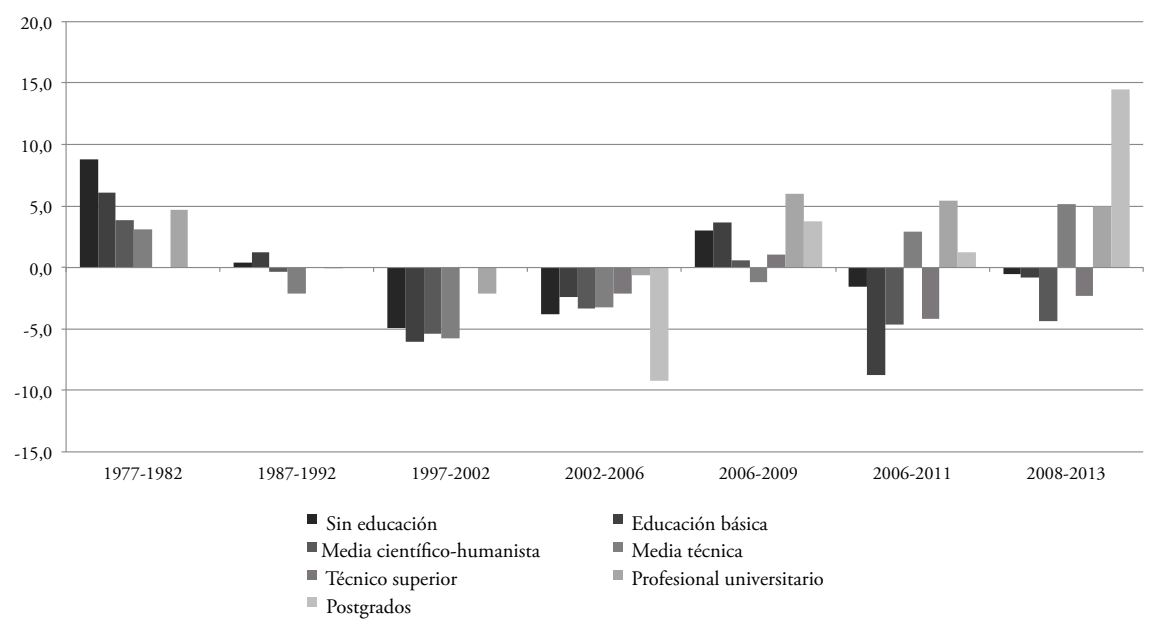

FUENTE ELABORACIÓN PROPIA A PARTIR DE BASES DE MICRODATOS DE CENSOS Y ENCUESTAS, PROCESAMIENTOS ESPECIALES

Sobre el efecto de la migración en la composición por sexo, edad y educación del AMGs

\section{Efecto sobre la relación de masculinidad}

De la figura 6 puede concluirse que hasta mediados de la década de 2000, la migración interna tendía a reducir la relación de masculinidad del Amgs. Pero desde mediados de la década de 2000 este efecto tendió a desaparecer y hasta se revirtió, por cuanto, de acuerdo con la última CASEN, la migración interna tendió a masculinizar Santiago.

Respecto de la magnitud de los efectos, el más significativo fue alcanzado en el quinquenio 1977-1982 y fue de casi -1,5\%. Esto significa que la migración, de manera directa y exclusiva, redujo en un 1,5\% el nivel de la relación de masculinidad del AmGs. Si bien no parece un cambio muy significativo en una primera mirada, un examen demográfico más detenido releva que sí se trata de un efecto importante, por cuanto cambios significativos de la relación de masculinidad tardan tiempo en producirse (United Nations, 2015), y cuando son rápidos es porque hay eventos atípicos, sobre todo en materia de mortalidad diferencial según sexo, como conflictos armados.

Un insumo clave para la discusión de este hallazgo es proporcionado por los otros dos componentes de la figura 6, a saber, los efectos absolutos de la inmigración y la emigración. ${ }^{7}$ A diferencia de lo expuesto en la literatura sobre América Latina

7 Se advierte que el efecto relativo y los efectos absolutos tienen métricas diferentes, ya que el efecto relativo se expresa en puntos porcentuales de cambio del atributo respecto del "contrafactual", mientras que los efectos absolutos de la inmigración y la emigración se expresan como cambios en el valor del atributo debidos a la inmigración y la emigración. 
(CEPAL, 2012; Rodríguez \& Busso, 2009), el efecto de la inmigración ha tenido un comportamiento errático y más bien masculinizador, porque en la mayoría de los quinquenios incluidos en el periodo examinado tuvo un valor positivo, es decir, aumentó la relación de masculinidad. De hecho, el efecto feminizador del periodo 1977-1982 se debió a que ambos efectos se sumaron por ser ambos reductores de la relación de masculinidad.

FIGURA 6 | Efecto relativo de la migración neta y efecto absoluto de la inmigración y de la emigración sobre la relación de masculinidad. AMGS, población de 5 años y más

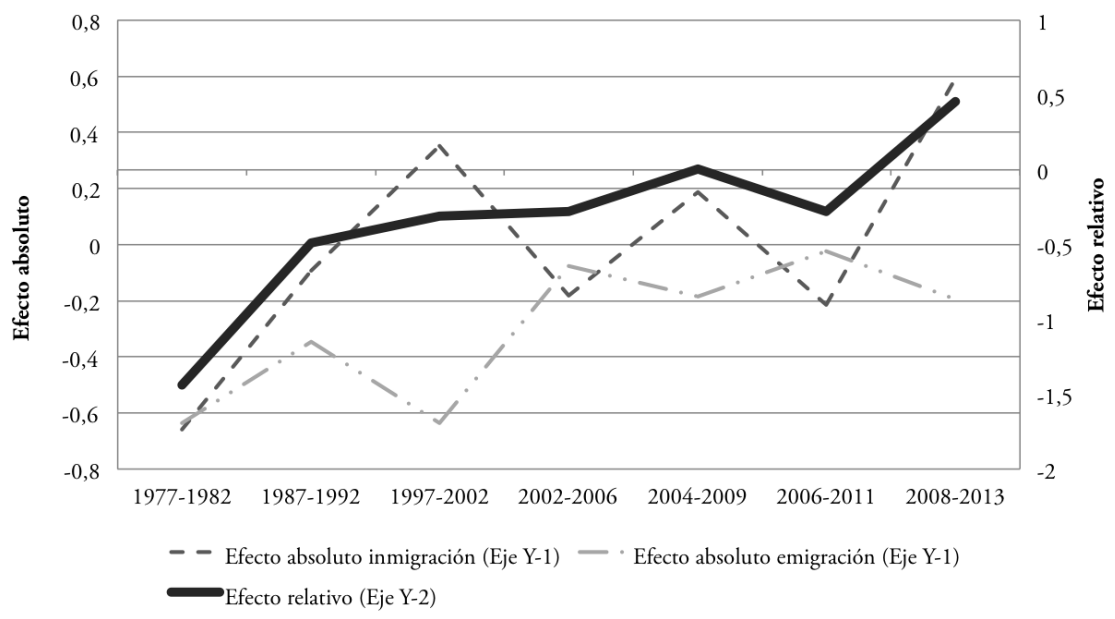

FUENTE ELABORACIÓN PROPIA A PARTIR DE BASES DE MICRODATOS DE CENSOS Y ENCUESTAS, PROCESAMIENTOS ESPECIALES

\section{Efecto sobre la composición por edad}

La figura 7 muestra que el principal efecto de la migración sobre la estructura etaria del AMGs es rejuvenecerla, en el sentido de aumentar la representación relativa de los jóvenes (15-29) y reducir al mismo tiempo la de todos los otros grandes grupos de edad. Este efecto no solo se mantiene durante todo el periodo de referencia, sino que luego de un descenso en la década de 1990 y principios de la década de 2000, recupera vigor, de acuerdo con las dos últimas CASEN, aunque sin llegar a los niveles observados en el primer quinquenio del periodo examinado (1977-1982).

Los niveles del efecto relativo de la migración neta son más elevados que los observados en el caso de la relación de masculinidad. Se acercan al $4 \%$ en el quinquenio de 1977-1982 y en ningún quinquenio del periodo examinado bajan del $1 \%$. Dado que la proporción que representaban los jóvenes en el AMGs (sobre la población de 5 años y más incluida en la matriz) hubiera sido del orden de 33,6\% (contrafactual), el incremento en un 4\% de este valor implicó una representación observada de este grupos de 34,6\% (factual) en 1982. 
La ganancia de representación relativa de los jóvenes ha sido a expensas de la representación relativa de los otros grandes grupos de edad, que durante todo el periodo (con la excepción del grupo de 60 años y más en el quinquenio 20042009) reducen su porcentaje dentro de la población de 5 años y más, por efecto de la migración. Ciertamente, en directa concomitancia con la merma del efecto incrementador del porcentaje de jóvenes, sobre todo entre mediados de la década de 1990 y mediados de la década de 2000, se atenúa también el efecto reductor del porcentaje de los otros grupos, pero, aun así, la última medición basada en la CASEN 2013 mantiene el patrón de un grupo que aumenta su proporción (15-29), contra los otros tres que la reducen.

FIGURA 7 Efecto relativo de la migración neta sobre la estructura de la población por grandes grupos de edad, población de 5 años y más

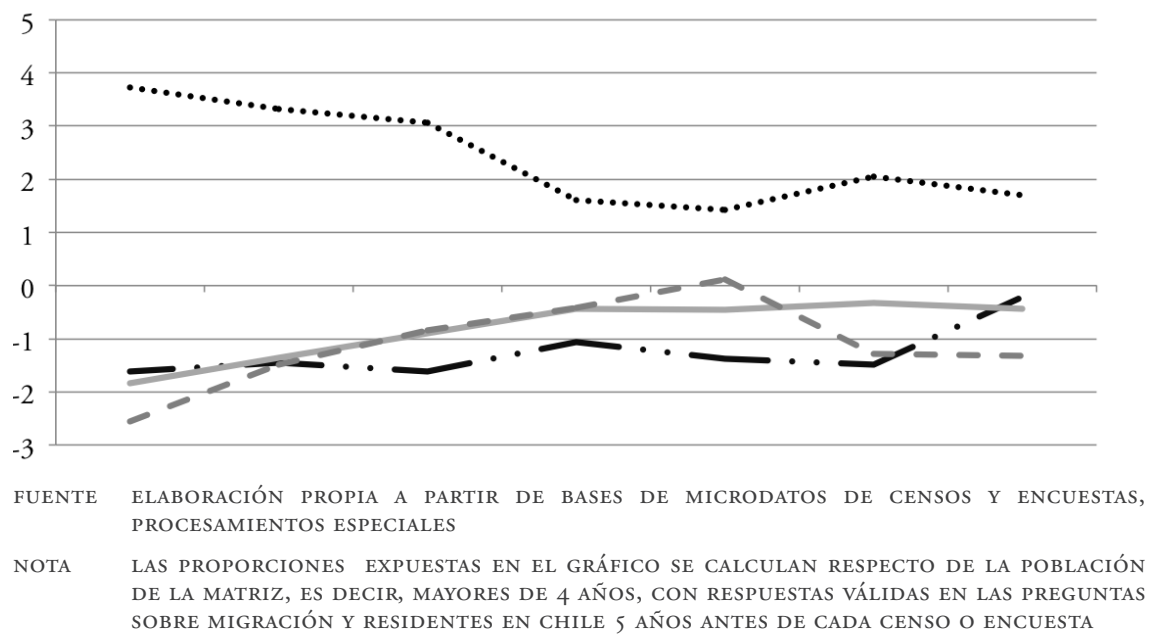

\section{Efecto sobre la composición por nivel educativo}

Finalmente, en la figura 8 se presenta una síntesis de los resultados del efecto de la migración sobre la composición educativa del AMGs. Las categorías educativas ya usadas en el análisis del atractivo migratorio según nivel educativo se mantienen y, desde luego, los resultados de este atractivo se expresan ahora en el efecto de la migración sobre la composición educativa del AMGs.

Para facilitar la apreciación visual del efecto de la migración sobre la composición educativa, en la figura 8 se exponen solo tres categorías educativas, que conforman el grueso de la población. Nótese que se presentan dos grupos etarios -25-29 años y 30-34 años- con los propósitos de: (i) cumplir con el supuesto de invariabilidad del atributo en el periodo de referencia que requiere la aplicación del procedimiento; y (ii) controlar el efecto distorsionador de la selectividad etaria de los migrantes sobre las diferencias educativas entre migrantes y no migrantes.

Los patrones y tendencias que se aprecian en la figura 8 no son tan estilizados como en el caso del efecto sobre la estructura por edad, pero de cualquier manera 
es evidente que la migración beneficia al AMGs en materia educativa, porque: (i) contribuye a ensanchar la representación de recursos humanos calificados (con formación universitaria); y (ii) tiende a reducir la representación de los recursos humanos con baja calificación (educación básica). Este efecto incrementador de los recursos humanos calificados presenta una tendencia al alza, en particular en el grupo de 30 a 34 ańos de edad. De hecho, en el quinquenio de 1977-1982 la situación era distinta: la migración tendía a reducir el nivel educativo del AMGs, pues los universitarios reducían su representación relativa debido a la migración (sobre todo en el grupo de edad de 30 a 34 años) y, en cambio, el grupo de las personas sin educación ${ }^{8}$ tendía a aumentar su representación.

FIGURA 8 Efecto relativo de la migración neta sobre la proporción de población con educación básica, media y universitaria. AMGs, población de 25 a 29 años y de 30 a 34 ańos

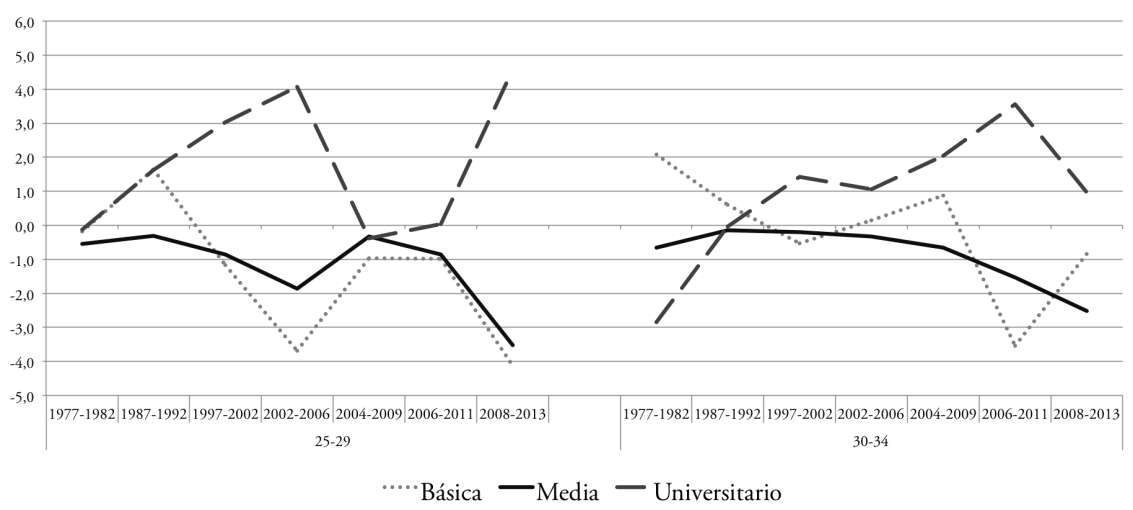

FUENTE ELABORACIÓN PROPIA A PARTIR DE BASES DE MICRODATOS DE CENSOS Y ENCUESTAS, PROCESAMIENTOS ESPECIALES

\section{Discusión y desafíos de investigación y de política}

La primera hipótesis del documento, que apunta a la continuación del atractivo migratorio del AMGs en virtud de sus ventajas comparativas respecto de los otros dos ámbitos considerados en este estudio (resto de la RM y resto del país), se descarta con la evidencia recabada. En efecto, no obstante las condiciones socioeconómicas relativamente más favorables del AMGs, esta ha sido básicamente expulsora de población en las décadas de 1990 y 2000.

Con todo, el atractivo del "resto de la $\mathrm{RM}^{\text {" }}$ sugiere que la condición expulsora del AMGs pudiera cambiar con una definición más amplia del AMGs que considere a algunas comunas de la RM excluidas de la definición usada. Más aún, de acuerdo con la CASEN 2013, al agrupar el AMGS con el resto de las comunas de la RM, lo que permite

$8 \quad$ No aparece en la figura 8, pero está disponible a solicitud.

9 Datos no presentados en este documento, pero previstos para una publicación próxima y disponibles a solicitud. 
reconstituir la RM como entidad de referencia, el saldo migratorio de la RM con el resto de las regiones del país es positivo, con lo cual no habría desconcentración demográfica por migración a escala regional, a diferencia de lo mostrado por las CASEN de 2011 y de 2006, y por el censo de 2002. Así las cosas, será necesario esperar nuevas encuestas, y sobre todo el censo abreviado de 2017, para realizar una nueva evaluación de esta hipótesis. En esta línea, cabría considerar para el futuro una definición ampliada del AMGS, para ofrecer insumos empíricos novedosos al debate metodológico.

Respecto del atractivo migratorio según sexo y el efecto de la migración sobre la composición por sexo del AMGs, los resultados permiten concluir que el AMGs ha dejado de ser una zona particularmente atractiva para las mujeres, y de hecho en los últimos años la migración neta de los hombres ha sido mayor (o menos negativa). Se acaba, así, una etapa en la cual la migración interna fue decisiva para la feminización del AmGs. Y en el horizonte no hay indicios de procesos que pudieran revertir esta situación, por lo cual lo más probable es que el efecto de la migración sobre la composición por sexo del AmGs tienda a la neutralidad.

Sobre el atractivo migratorio según grandes grupos de edad y el efecto de la migración sobre la composición por grandes grupos de edad del AMGs, los resultados comprueban, en primer lugar, el pertinaz atractivo que el AMGs ejerce para los jóvenes, es decir, para la población que tiene entre 15 y 29 años de edad. Este hallazgo no debe considerarse distintivo ni menos exclusivo del AmGs. Parece ser relativamente generalizado a escala global (Pacione, 2009), y sobre todo es claramente la norma en América Latina (CEPAL, 2012; Rodríguez \& Busso, 2009). La explicación detallada de esta regularidad escapa al alcance de este trabajo, pero si se revisa nuevamente la figura 1, se advierte la superioridad del AMGs en materia de oferta laboral y, sobre todo, de oferta educativa para los jóvenes. No obstante la reconocida ampliación de la cobertura de todos los niveles del sistema educativo chileno, ${ }^{10}$ la oferta de mayor calidad en la educación secundaria, así como una fracción significativa de la oferta general y de mayor calidad del nivel universitario, aún están sobrerrepresentadas en el AMGS, tal como se aprecia, con relación a la oferta general, en la figura 9.

De hecho, virtualmente no existen universidades en ciudades pequeñas, e incluso en algunas de las quince regiones del país hace poco no había universidad estatal. ${ }^{11}$ Más fuerte, incluso, puede ser la oferta de posgrados: en la figura 1 queda en evidencia que la mayor ventaja del AMGs respecto del promedio nacional en materia educativa es en la tasa de asistencia del grupo de 25 a 29 ańos, con mayores probabilidades de estar realizando posgrados. Otro tanto ocurre en materia laboral, ya que la participación laboral juvenil en el AMGS es mayor que el promedio nacional y el nivel de desempleo es inferior.

Este atractivo tan marcado que ejerce el AMGS para los jóvenes redunda en un efecto sistemático y persistente de la migración interna sobre la estructura por edad del AMGs, cual es el ensanchamiento de la representación de este grupo etario a

10 Véase http://observatorio.ministeriodesarrollosocial.gob.cl/casen/casen_def_educacion.php

11 Carencia recientemente subsanada (agosto de 2015) por la aprobación de la Ley Núm. 20842, Ministerio de Educación. (http://www.leychile.cl/Navegar?idNorma=1080237). 
expensas de todos los otros. Al rejuvenecer el AMGs, refuerza y extiende la vigencia del denominado bono demográfico en el AMGs, lo que ha sido verificado en varias otras áreas metropolitanas de la región (Rodríguez, 2013b).

FIGURA 9 | Región Metropolitana de Santiago, 2002-2015: evolución del porcentaje que representa en la matrícula de educación superior y en la población de 18 a 25 años

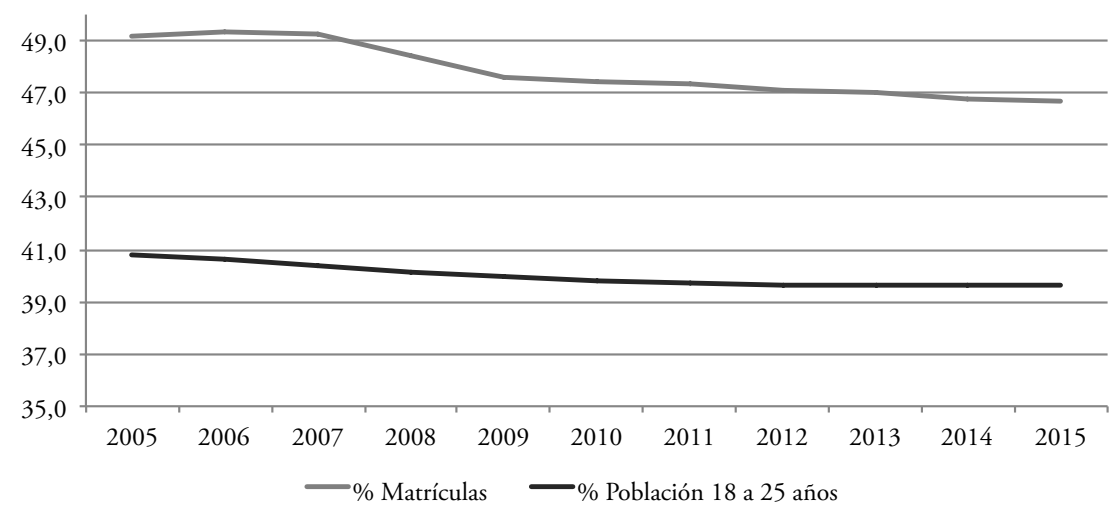

FUENTE MATRÍCULAS: CONSEJO NACIONAL DE EDUCACIÓN. TENDENCIAS ÍNDICES 2O I 5, WWW.CNED.CL/PUBLIC/ SECCIONES/SECCIONINDICESPOSTULANTES/OTRASESTADISTICAS/TENDENCIAS_INDICES_2OI 5.PDF; POBLACIÓN: WWW.INE.CL/CANALES/CHILE_ESTADISTICO/FAMILIAS/DEMOGRAFICAS_VITALES.PHP

Por cierto, este efecto no ha impedido que la proporción que representan los jóvenes dentro de la población de 5 años y más se reduzca durante el periodo examinado en directa relación con el proceso de transición demográfica. De hecho, esta proporción se redujo de $34,6 \%$ a $27 \%$ en el periodo examinado, ${ }^{12}$ pero la reducción hubiera sido aún mayor de no ser por el efecto en el sentido opuesto de la migración neta.

Cualquiera sea el caso, resulta un efecto ventajoso, en principio, tanto por el bono demográfico como por el dinamismo, empuje y creatividad asociados a la población juvenil.

En lo que atañe al atractivo migratorio según niveles educativos y el efecto de la migración sobre la composición por niveles educativos, se verifica que, a diferencia de lo observado en la mayor parte de las ciudades de América Latina (Rodríguez, 2013b), en el AMgs la migración interna contribuye a incrementar los recursos humanos calificados, retroalimentando sus ventajas comparativas en este plano respecto del resto del país.

Estos resultados ofrecen una nueva mirada sobre el controversial asunto de la concentración de población, recursos y poder en el AMGs. Claramente, el AMGS parece beneficiarse de la migración y esta contribuye a fortalecer su posición aventajada respecto del resto del país. Los análisis que solo se concentran en la cantidad de población que atrae o que sale de allí resultan estrechos para evaluar el aporte de la migración al desarrollo del AMGs. El hecho de que su atractivo sea sobresaliente para

12 Datos no presentados en el trabajo, pero disponibles a solicitud. 
los jóvenes y las personas de mayor educación es sintomático de las oportunidades que concentra el AMGs: formación de nivel superior, empleos mejor remunerados y plazas laborales más cotizadas. De esta manera, una agenda desconcentradora debiera incluir estos aspectos, incentivando que tanto los planteles de mejor calidad del sistema de educación superior, como los centros directivos de empresas públicas y privadas se distribuyan de una forma menos concentrada en el AMGs.

Se trata de una línea de acción desafiante, por los beneficios, y también la inercia y el denominado path dependence, que entraña esta concentración para las instituciones referidas. En tal sentido, debe ser evaluada cuidadosamente para lograr compatibilizar, en la medida de lo posible, ambos objetivos: el de desconcentración para el desarrollo y la equidad regional, y el de eficiencia para el desarrollo institucional y productivo nacional. Incluso más, tales iniciativas deben tener en cuenta un conjunto de fuerzas que se mueven en sentido contrario al desplazamiento de población hacia las regiones, tales como las nuevas modalidades de jornada laboral implementadas en las regiones mineras, en particular el sistema de turnos semanales; el aumento de la conmutación laboral interregional producto de la mejora del transporte; la expansión del teletrabajo y la histórica práctica del trabajo estacional en la regiones agrícolas. En suma, avanzar hacia una desconcentración de las empresas, incluyendo las del Estado, y de los trabajadores en general, requiere de un esfuerzo nacional y una política de Estado amplia y multisectorial.

\section{Referencias bibliográficas}

Alberts, J. (1977). Migración hacia áreas metropolitanas de América Latina: un estudio comparativo. Serie E, Celade, n. ${ }^{\circ}$ 117. Santiago, Chile: Centro Latinoamericano y Caribeño de Demografía (Celade). http://repositorio.cepal.org/handle/11362/7795

Amin, A. (Ed.). (2001). Post-Fordism: A reader. Oxford: Blackwell/John Wiley \& Sons. doi: $0.1002 / 9780470712726$

Atienza, M. \& Aroca, P. (2012). Concentración y crecimiento en Chile: una relación negativa ignorada. EURE, 38(114), 257-277. http://dx.doi.org/10.4067/S025071612012000200010

Bell, M. \& Salut, M. (2009). Cross-National Comparisons of Internal Migration. Human Development Research Paper 2009/30. United Nations Development Programme (UNDP). http://hdr.undp.org/sites/default/files/hdrp_2009_30.pdf

Berg, L., Drewett, R., Klaasen, L. H., Rossi, A. \& Vijverberg, C. H. T. (1982). Urban Europe: A study of growth and decline. The Costs of Urban Growth (сURB) Project, vol. 1. Oxford: Pergamon Press.

Brenner, N. (2013). Tesis sobre la urbanización planetaria. Nueva Sociedad, (243), 38-66. http://nuso.org/articulo/tesis-sobre-la-urbanizacion-planetaria/

Camisa, Z. (1972). Efecto de la migración en el crecimiento y la estructura de la población de las ciudades de la América Latina. Serie C, Celade, $n .{ }^{\circ} 139$. Santiago, Chile: Centro Latinoamericano y Caribeño de Demografía (Celade). http://repositorio.cepal.org/handle/11362/7915 
Campolina, C. (1994). Polygonized development in Brazil: Neither decentralization nor continued polarization. International Journal of Urban and Regional Research, 18(2), 293-314. doi: 10.1111/j.1468-2427.1994.tb00267.x

Chávez, A. M., Rodríguez, J., Acuña, M., Barquero, J., Macadar, D. \& Sobrino, J. (2013). Nouvelles tendances de la migration métropolitaine en Amérique Latine: est-ce que les aires métropolitaines gagnent ou perdent population à cause de la migration interne? Artículo presentado en xxvii Conference of the International Union for the Scientific Study of Population (IUSSP), Busan, República de Corea, 08/2013. http://bit.ly/2bCAiM9

Comisión Económica para América Latina y el Caribe, de las Naciones Unidas (CEPAL). (2012). Población, territorio y desarrollo sostenible (LC/L.3474 (CEP.2/3)). Santiago, Chile: CEPAL. http://www.cepal.org/celade/noticias/paginas/0/46070/2012-96-poblacion-web.pdf

Comisión Económica para América Latina y el Caribe, de las Naciones Unidas (CEPAL). (2015). Panorama del desarrollo territorial en América Latina y el Caribe, 2015: pactos para la igualdad territorial (LC/w.67I). Santiago, Chile: CEPAL. http://repositorio.cepal.org/ handle/11362/39223

Cunha, J. M. (2015). Dinâmica demográfica e migratória 1991-2010: realidades e mitos. En E. Marques (Org.), A metrópole de São Paulo no século XXI: espaços, heterogeneidades e desigualdades (pp. 107-146). São Paulo: Editora unesP (Universidade Estadual Paulista).

Cunha, J. M. \& Rodríguez, J. (2010). Crecimiento urbano y movilidad en América Latina. Revista Latinoamericana de Población, 3(4-5), 27-64. http://www.redalyc.org/articulo. oa?id=323827368003

Daher, A. (2003). Regiones-commodities: Crisis y contagio en Chile. EURE, 29(86), 89-108. http://dx.doi.org/10.4067/S0250-71612003008600005

De Mattos, C. (2010). Globalización y metamorfosis metropolitana en América Latina: de la ciudad a lo urbano generalizado. Revista de Geografia Norte Grande, (47), 81-104. http://dx.doi.org/10.4067/S0718-34022010000300005

De Mattos, C., Fuentes, L. \& Link, F. (2014). Tendencias recientes del crecimiento metropolitano en Santiago de Chile. ¿Hacia una nueva geografía urbana? Revista INVI, 29(81), 193219. http://www.revistainvi.uchile.cl/index.php/Invi/article/view/837/1148

De Mattos, C. \& Hidalgo, R. (Eds.) (2007). Santiago de Chile: Movilidadespacialy reconfiguración metropolitana. Santiago, Chile: Pontificia Universidad Católica de Chile, Instituto de Estudios Urbanos, Serie GEOlibros n. ${ }^{\circ} 8$.

Dureau, F., Lulle, T., Souchaud, S. \& Contreras, Y. (Dirs.). (2014). Mobilités et changement urbain. Bogotá, Santiago et São Paulo. Rennes: Presses Universitaires de Rennes.

Elizaga, J. (1970). Migraciones a las áreas metropolitanas de América Latina. Serie E, Celade, n. ${ }^{\circ}$ 6. Santiago, Chile: Centro Latinoamericano de Demografía (Celade). http:// repositorio.cepal.org/handle/11362/7337

Elizaga, J. (1972). Migraciones interiores, el proceso de urbanización, movilidad social. Serie A, Celade, n. ${ }^{\circ}$ 117. Santiago, Chile: Centro Latinoamericano de Demografía (Celade). http://repositorio.cepal.org/handle/11362/7987

Elizaga, J. \& Macisco, J. (1975). Migraciones internas. Teoría, método y factores sociológicos. Serie E, Celade, n. ${ }^{\circ}$ 19. Santiago, Chile: Centro Latinoamericano de Demografía (Celade). http://repositorio.cepal.org/handle/11362/7611 
Fondo de Población de las Naciones Unidas (UNFPA). (2007). Estado de la población mundial, 2007. Liberar el potencial del crecimiento urbano. Nueva York: UNFPa. https://www. crin.org/en/docs/unfpa_07.pdf

Fuentes, L. \& Sierralta, C. (2014). Santiago de Chile, ¿ejemplo de una reestructuración capitalista global? EURE, (30)91, 7-28. http://dx.doi.org/10.4067/S0250-71612004009100002

Fujita, M., Krugman P. \& Venables, A. (2000). The spatial economy: Cities, regions, and international trade. Cambridge, MA: The міт Press.

Galetovic, A. \& Jordan, P. (2006). Santiago: ¿̇dónde estamos?, ¿hacia dónde vamos? Revista Estudios Públicos, (101), 87-146. http://www.cepchile.cl/santiago-donde-estamoshacia-donde-vamos/cep/2016-03-04/093914.html

Geyer, M. \& Kontuly, T. (1993). A theoretical foundation for the concept of differential urbanization. International Regional Science Review, 15(2), 157-177. doi: $10.1177 / 016001769301500202$

Gilbert, A. (1996). The mega-city in Latin America. Tokio/Nueva York/París: United Nations University Press. http://archive.unu.edu/unupress/unupbooks/uu23me/uu23me00.htm

Glaeser, E. (2011). Cities, productivity, and quality of life. Science, 333(6042), 592-594. doi: $10.1126 /$ science. 1209264

Greenwood, M. (1997). Internal migration in developed countries. En M. R. Rosenzweig (Ed.), Handbook of Population and Family Economics, Vol. 1, Part. B, 647-720. Ámsterdam: Elsevier. http://dx.doi.org/10.1016/S1574-003X(97)80004-9

Hall, P. (1996). Ciudades del mañana. Historia del urbanismo en el siglo XX. Barcelona: Ediciones del Serbal.

Henderson, J. (2000). How urban concentration affects economic growth. Policy Research Working Paper 2326. Washington, DC: The World Bank. http://dx.doi.org/10.1596/1813$9450-2326$

Henderson, J. (2003). The urbanization process and economic growth: The so-what question. Journal of Economic Growth, 8(1), 47-71. doi: 10.1023/A:1022860800744

Ingram, G. (1998). Patterns of metropolitan development: What have we learned? Urban Studies, 35(7), 1019-1035. doi: 10.1080/0042098984466

Martine, G., McGranahan, G., Montgomery, M. \& Fernández-Castilla, R. (Eds.) (2008). The New Global Frontier: Cities, Poverty and Environment in the $21^{15 t}$ Century. Londres: IIED/ UNFPA and Earthscan Publications. http://www.freebookspaindownload.org/edicionespecial/the-new-global-frontier

Organisation for Economic Co-operation and Development (OECD). (2012). Compact city policies: A comparative assessment. oecd Green Growth Studies. OeCD Publishing. http://dx.doi.org/10.1787/9789264167865-en

Pacione, M. (2009). Urban Geography. A global perspective. Nueva York: Routledge.

Rodríguez, J. (2002). Distribución espacial de la población de América Latina y el Caribe: Tendencias, interpretaciones y desafios para las politicas públicas. Serie Población y Desarrollo, n. 32 (Lc/L.I83 I-P). Santiago, Chile. Comisión Económica para América Latina y el Caribe (CEPAL). http://repositorio.cepal.org/bitstream/handle/11362/7170/1/S02121008_es.pdf

Rodríguez, J. (2013a). La migración interna en las grandes ciudades en América Latina: efectos sobre el crecimiento demográfico y la composición de la población. Notas de Población, 40(96), 53-104 (LC/G.2573-P). Santiago, Chile: Comisión Económica para América Latina y el Caribe (CEPAL). http://repositorio.cepal.org/handle/11362/12909 
Rodríguez, J. (2013b). How is internal migration reshaping metropolitan populations in Latin America? New methodologies and new evidence. Artículo presentado en la XXVII Conference of the International Union for the Scientific Study of Population (IUSSP), Busan, República de Corea, 08/2013. http://bit.ly/2aW1daJ

Rodríguez, J. \& Busso, G. (2009). Migración interna y desarrollo en América Latina entre 1980 y 2005. Un estudio comparativo con perspectiva regional basado en siete paises. Libros de la CEPAL, n. ${ }^{\circ} 102$ (LC/G.2397-P). Santiago, Chile: Comisión Económica para América Latina y el Caribe (CEPAL). http://repositorio.cepal.org/bitstream/ handle/11362/2541/1/S0800671_es.pdf

Rodríguez, J. \& Espinoza, D. (2012). Recuperación del atractivo migratorio metropolitano en el periodo 2004-2009: ¿factores exógenos o endógenos? Revista de Geografia Norte Grande, (51), 95-113. http://dx.doi.org/10.4067/S0718-34022012000100006

Rodríguez, J. \& González, D. (2006). Redistribución de la población y migración interna en Chile: continuidad y cambio según los últimos cuatro censos nacionales de población y vivienda. Revista de Geografia Norte Grande, (34), 7-28. http://dx.doi.org/10.4067/ S0718-34022006000100002

Sabatini, F. (1991). Santiago: tendencias y posibilidades de desconcentración de la industria en la macro región central. EURE, 27(52/53), 75-86. http://www.eure.cl/index.php/ eure/article/view/1076

Sabatini, F. \& Wormald, G. (2008). Santiago, Chile bajo la nueva economía (1980-2000). Crecimiento, modernización y oportunidades de integración social. En A. Portes, B. Roberts \& A. Grimson (Eds.), Ciudades latinoamericanas: un análisis comparativo en el umbral del nuevo siglo (pp. 217-298). México, DF: Miguel Ángel Porrúa.

Sassen, S. (1991). The Global City. Princeton: Princeton University Press.

Sassen, S. (2007). El reposicionamiento de las ciudades y regiones urbanas en una economía global: ampliando las opciones de políticas y gobernanza. EURE, 33(100), 9-34. http://dx.doi.org/10.4067/S0250-71612007000300002

United Nations (2008). United Nations Expert Group Meeting on Population Distribution, Urbanization, Migration and Development (ESA/p/wr.206). Nueva York: United Nations, Department of Economic and Social Affairs, Population Division [Online]. www.un.org/esa/population/meetings/EGM_PopDist/EGM_PopDist_Report.pdf

United Nations (2015). World Urbanization Prospects: The 2014 Revision (st/ESA/sER.A/366). Nueva York: United Nations, Department of Economic and Social Affairs, Population Division. https://esa.un.org/unpd/wup/Publications/Files/WUP2014-Report.pdf

Villa, M. \& Rodríguez, J. (1997). Dinámica sociodemográfica de las metrópolis latinoamericanas durante la segunda mitad del siglo xx. Notas de Población, 25(65), 17-110 (LC/ DEM/G. I77). http://repositorio.cepal.org/handle/11362/12518

Williamson, J. (1965). Regional inequality and the process of national development: A description of the patterns. Economic Development and Cultural Change, $13(4-$ Part 2), 1-84. 


\section{Anexo técnico \\ La matriz de indicadores de flujo y la aplicación del procedimiento}

La matriz de más abajo es novedosa aunque no nueva, pues ya se ha expuesto en publicaciones previas (Rodríguez, 2013a, 2013b; Rodríguez \& Busso, 2009). Su novedad estriba en que los casilleros no contienen población, sino un indicador. Se trata de indicadores de los flujos (o corrientes) migratorios, incluyendo la diagonal, que en rigor no es un flujo (se trata de los no migrante). Para obtener esta matriz hay que procesar los microdatos (de censos, encuestas o registros continuos de población), porque hasta ahora no se publican ni en medios impresos ni electrónicos. El procesamiento requerido varía según el tipo de indicador y está descrito con detalle en la literatura ya referida.

Ahora bien, el procedimiento para estimar el efecto de la migración sobre la composición de la población solo usa los marginales (o totales) de la matriz y la diagonal. El marginal columna en la matriz de más abajo (columna 1) corresponde al porcentaje de población joven, ${ }^{13}$ es decir, entre 15 y 29 ańos, que reside en cada una de las tres entidades de la matriz (AMGs, resto de la Región Metropolitana, resto del país) al momento del censo o la encuesta y por eso se denomina total actual o factual. En cambio, el marginal fila (columna 2) corresponde al porcentaje que hubiese existido en estas entidades si la población no se hubiese movido de la entidad donde vivía cinco años antes; es decir, si no hubiese habido migración en el periodo de referencia; por ello se denomina contrafactual. Con estas dos columnas se efectúan los cálculos en las columnas que siguen, que se enumeran de 3 a 6 y que tienen indicados los cálculos que contienen. La intuición básica del procedimiento es que la diferencia entre el valor factual y el contrafactual se debe exclusivamente a la migración, pues en esta población no hay nacimientos ni defunciones ni ningún otro tipo de evento que pueda afectar la estructura etaria. Por ello, el efecto absoluto de la migración corresponde a la resta del valor factual y el contrafactual, y el efecto relativo al resultado de esta resta dividido por el valor contrafactual. Finalmente, los efectos de la inmigración y la emigración pueden ser obtenidos como la resta entre el valor factual y el valor de la diagonal, y la resta entre el valor de la diagonal y el valor factual, respectivamente. Las fórmulas en lenguaje algebraico y matricial pueden encontrarse en Rodríguez (2013a, 2013b).

El procedimiento tiene supuestos -en particular el de invariabildad temporal del atributo-, lo que limita su aplicación a las variables que los cumplen. Además, por provenir de una matriz de migración obtenida con la consulta por fecha fija, hay pérdida de migrantes; y si los migrantes no captados difieren de los sí captados, la estimación puede estar sesgada. Lamentablemente, no hay modo de comparar los migrantes captados con los no captados. Una prevención básica es que el cambio en el atributo producido por la migración no corresponde al cambio efectivo del

13 Nota: Los porcentajes se calculan respecto de la población de la matriz, es decir, mayores de 4 ańos con respuestas válidas en las preguntas sobre migración y residentes en Chile 5 años antes de cada censo o encuesta (o el periodo definido en la fuente, ya que la CASEN 2006 usó una fecha fija diferente a 5 ańos, específicamente en torno a 4,5 ańos, para cubrir el periodo entre el censo de 2002 y la fecha de realización de la CASEN 2006). 
atributo, porque en el periodo de referencia este puede haber cambiado por otros factores demográficos (el diferencial de crecimiento vegetativo según el atributo) y sociales (movilidad social).

Para concluir, la interpretación de los resultados es que el AMGs tenía en 2013 un porcentaje de jóvenes de 26,97, el que habría sido de 26,52 en ausencia de migración. Por tanto, la migración elevó en 0,45 puntos porcentuales el porcentaje de jóvenes, lo que significa un aumento del 1,69\% de este porcentaje (respecto del que habría tenido sin migración). Casi todo el aumento (0,43 de los 0,45 puntos porcentuales) se debe a inmigración, porque los jóvenes están sobrerrepresentados entre los inmigrantes respecto de los no migrantes. 


\begin{tabular}{|c|c|c|c|c|c|c|}
\hline \multirow{4}{*}{ 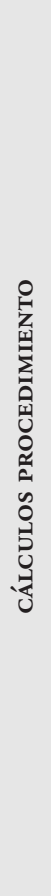 } & 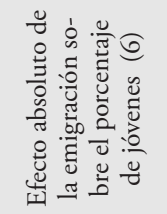 & 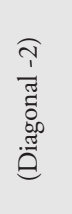 & 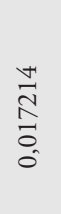 & 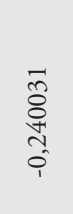 & 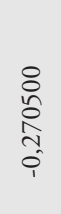 & \multirow{4}{*}{ 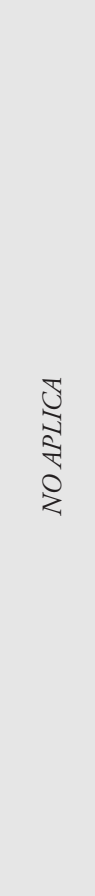 } \\
\hline & 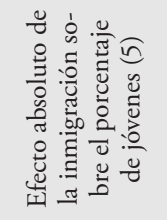 & 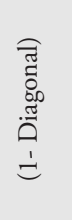 & \begin{tabular}{l}
$\stackrel{0}{+}$ \\
$\stackrel{+}{+}$ \\
\multirow{2}{+}{} \\
0
\end{tabular} & $\begin{array}{l}\text { } \\
\hat{\curvearrowright} \\
\infty \\
0 \\
0\end{array}$ & $\begin{array}{l}\hat{n} \\
0 \\
0 \\
0\end{array}$ & \\
\hline & 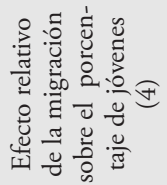 & $\underset{\mathfrak{d}}{\widehat{d}}$ & $\stackrel{\text { }}{-}$ & $\begin{array}{l}n \hat{n} \\
\hat{i}\end{array}$ & $\frac{8}{1}$ & \\
\hline & 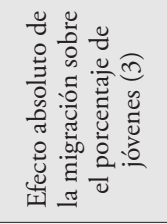 & $\stackrel{\widehat{I}}{=}$ & $\begin{array}{l}\text { ff } \\
\text { of }\end{array}$ & $\frac{n}{i}$ & $\begin{array}{l}\text { ते } \\
\text { n̂. }\end{array}$ & \\
\hline \multirow{5}{*}{ 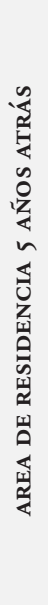 } & \multicolumn{2}{|c|}{ 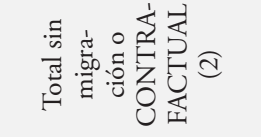 } & 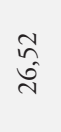 & $\begin{array}{l}\text { जे } \\
\hat{\sim}\end{array}$ & $\begin{array}{l}\overline{\hat{}} \\
\hat{\approx}\end{array}$ & 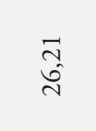 \\
\hline & \multicolumn{2}{|c|}{ 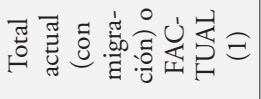 } & बे & $\begin{array}{l}\stackrel{\sim}{\sim} \\
\stackrel{\sim}{N}\end{array}$ & 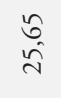 & $\begin{array}{l}\vec{\pi} \\
\hat{\text { ț }}\end{array}$ \\
\hline & \multicolumn{2}{|l|}{ 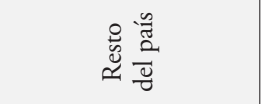 } & $\stackrel{n}{\stackrel{n}{q}}$ & $\begin{array}{l}\stackrel{\Xi}{0} \\
\stackrel{+}{m}\end{array}$ & $\begin{array}{l}\text { J゙ } \\
\stackrel{\sim}{v}\end{array}$ & $\begin{array}{l}\bar{\approx} \\
\hat{\approx}\end{array}$ \\
\hline & \multicolumn{2}{|c|}{ 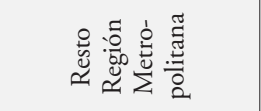 } & 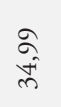 & $\begin{array}{l}\stackrel{n}{f} \\
\hat{N}\end{array}$ & $\begin{array}{l}n \\
\infty \\
\tilde{b}^{n}\end{array}$ & $\begin{array}{l}\hat{\sigma} \\
\hat{\sim}\end{array}$ \\
\hline & \multicolumn{2}{|l|}{$\sum_{<}^{0}$} & $\begin{array}{l}\stackrel{+}{n} \\
\text { in }\end{array}$ & 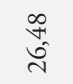 & $\begin{array}{l}\stackrel{0}{\infty} \\
\stackrel{\sim}{\sim}\end{array}$ & $\begin{array}{l}\tilde{N} \\
\hat{\tilde{N}} \\
\end{array}$ \\
\hline & \multirow{2}{*}{\multicolumn{2}{|c|}{ 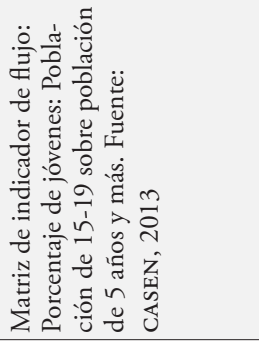 }} & $\frac{y}{4}$ & 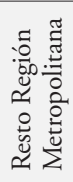 & 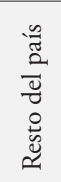 & 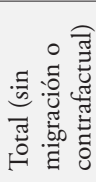 \\
\hline & & & \multicolumn{4}{|c|}{ 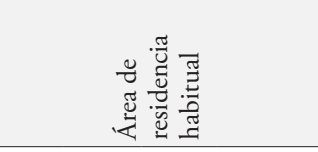 } \\
\hline
\end{tabular}

